\title{
A Rare Case of Burkholderia Pseudomallie Presenting as Brain Abscess
}

\author{
Allen S. Sibil, Suresh S. Kumar, and Alex Aiswariya
}

\section{ABSTRACT}

Rationale: Meiliodosis is an extremely rare condition in Neurosurgery which is caused by the gram negative organism Burkholderia pseudomallie. Burkholderia pseudomallei (also known as Pseudomonas pseudomallei) is a Gram-negative, bipolar, aerobic, motile rod-shaped bacterium. It is a soildwelling bacterium endemic in tropical and subtropical regions worldwide, particularly in Thailand and northern Australia. It infects humans and animals and causes the disease melioidosis. It is also capable of infecting plants. If treatment is below par or delayed, this infection can produce diverse clinical symptoms and result in death.

Patient Concerns: A 52 year old female, known case of Type 2 Diabetes mellitus on insulin, presented with complaints of a swelling in the left parieto-occipital region since 2 months \& headache since 1 month. She had a past history of a fall from scooter 7 months ago, which then was not radiologically imaged. Patient was, otherwise, conscious, oriented and had no focal neurological deficit.

Diagnosis: left parieto-occipital space occupying lesion confirmed by $\mathrm{Ct}$ brain and [mri + mrv] brain and culture and sensitivity of fluid aspirate showed moderate growth of burkholderia pseudomallie.

Interventions: Intravenous Ceftazidime [1 $\mathrm{g} / 12 \mathrm{~h}]$ was administered inhospital for 4 weeks (Intensive Phase) and Oral Cotrimoxazole (Trimethoprim + sulfomethoxazole) \{TAB.BACTRIM DS $\}$ twice daily was administered out-hospital (Maintenance Phase).

Patient underwent Left parieto-occipital abscess drainage and debridement [Pus sent for Culture and Sensitivity] - Abscess drainage and removal of irregular thinned out bone around the skull defect + Biopsies sent from small extradural collection near the skull defect.Intravenous Ceftazidime was continued and discharged with Oral Cotrimoxazole (Trimethoprim + sulfomethoxazole).

Keywords: Meiliodosis, Type 2 Diabetes mellitus, skull defect, Glasgow Coma Scale.

\section{INTRODUCTION}

Burkholderia pseudomallie -Bacterium profile: $B$. pseudomallei measures $2-5 \mu \mathrm{m}$ in length and $0.4-0.8 \mu \mathrm{m}$ in diameter and is capable of self-propulsion using flagella. The bacteria can grow in a number of artificial nutrient environments, especially betaine- and arginine-containing ones.

B. pseudomallei is not fastidious and grows on a large variety of culture media (blood agar, MacConkey agar, EMB, etc.). Ashdown's medium (or Burkholderia cepacia medium) may be used for selective isolation. Cultures typically become positive in 24 to 48 hours (this rapid growth rate differentiates the organism from $B$. mallei, which typically takes a minimum of 72 hours to grow). Colonies are wrinkled, have a metallic appearance, and possess an earthy odour. On Gram staining, the organism is a Gram-negative rod with a characteristic "safety pin" appearance (bipolar staining). On sensitivity testing, the organism appears highly resistant (it is innately resistant to many antibiotics including colistin and
Published Online: January 11, 2021

ISSN: $2593-8339$

DOI: $10.24018 /$ ejmed.2021.3.1.620

Dr. Sibil S. Allen *

Amala Institute of Medical Sciences,

Thrissur, Kerala, India.

(e-mail: allenlwysakid@gmail.com)

Dr. Kumar S. Suresh

Amala Institute of Medical Sciences,

Thrissur, Kerala, India

Dr. Alex Aiswariya

Amala Institute of Medical Sciences,

Thrissur, Kerala, India.

*Corresponding Author gentamicin) and that again differentiates it from B. mallei, which is in contrast, exquisitely sensitive to many antibiotics. For environmental specimens only, differentiation from the nonpathogenic $B$. thailandensis using an arabinose test is necessary (B. thailandensis is never isolated from clinical specimens).

Laboratory identification of $B$. pseudomallei can be difficult, especially in Western countries where it is rarely seen. The large, wrinkled colonies look like environmental contaminants, so are often discarded as being of no clinical significance. Colony morphology is very variable, and a single strain may display multiple colony types, so inexperienced laboratory staff may mistakenly believe the growth is not pure. The organism grows more slowly than other bacteria that may be present in clinical specimens, and in specimens from nonsterile sites, is easily overgrown. Nonsterile specimens should, therefore, be cultured in selective media (e.g., Ashdown's or B. cepacia medium). For heavily contaminated samples, such as faeces, a modified version of Ashdown's that includes norfloxacin, amoxicillin, and 
polymyxin B has been proposed. In blood culture, the BacT/ALERT MB system (normally used for culturing mycobacteria) by bioMérieux has been shown to have superior yields compared to conventional blood culture media.

\section{CASE REPORT}

A 52 year old female with a past history of a fall from scooter in February 2020 which then was not radiologically imaged, presented with complaints of a swelling in the left parieto-occipital region since 2 months \& headache since 1 month in September 2020. She is known case of Type 2 Diabetes mellitus on insulin. On examination Patient was conscious, oriented, and had normal speech, vision, and a full GCS (Glasgow Coma scale) score of 15. CT Brain (Plain + Contrast) and MRI Brain (Plain + contrast) with MR Venogram were done and is reported as shown and patient was admitted to Hospital. Pus aspiration of the scalp swelling was done under local anesthesia and pus sent for Culture \& sensitivity and AFB staining. C \& S report showed moderate of growth of Burkholderia pseudomallie. Intensive treatment with Intraveous Ceftazidime for was started for 4 weeks. Along the course in hospital patient developed nausea, vomiting and giddiness, which later subsided. A CT brain (Plain) was done and showed the findings as reported. Drainage of abscess and debridement of lesion was planned. On October 16 2020, patient underwent Left Parieto-occipital abscess drainage and debridement under GA and Aspirate sent for Culture \& sensitivity. Removal of irregular thinned out bone around the skull defect and Biopsies were sent from small extradural collection near the skull defect. Exposed necrotic tissue was removed using curette. Drain was also placed insitu and wound closed in layers. Post-operative period was uneventful. Daily dressing and cleaning of the scalp wound was done. Initial symptoms of headache nausea , vomiting and giddiness subsided and now the patient was symptomatically better, vitals stable, ambulant and ready to be discharged. After 4 weeks treatment with Intravenous Ceftazidime, patient was discharged with maintenaince phase antibiotic treatment with Oral Co-trimoxazole (Tab.BACTRIM DS) twice daily with regular weekly follow up consultations (maintenance treatment was planned for 3-6 months).

\section{RADIOLOGICAL IMAGING REPORTS}

\section{A. MRI-Brain + MR Venogram (Plain + Contrast) Report}

A moderately defined lesion with thick enhancing walls noted in the scalp posteriorly with intracranial extension into left parietal lobe. D/d - Necrotic mass - suggested Histopathology correlation.

Absence of normal flow void noted in the adjacent superior sagittal sinus suggestive of thrombosis.

CT-Brain (Plain + Contrast): Thrombus in posterior aspect of superior sagittal sinus. Few loculated peripherally enhancing collections with mild perilesional edema involving the left parasagittal parietal lobe with extracranial thick walled peripherally enhancing component involving the scalp in posterior parietal region - possibly abscess (decreased in size compared to previous CT dated 16/09/2020 and 7/10/2020).

\section{PATHOGENICITY MECHANISM \& VIRULENCE FACTORS}

B. pseudomallei is an opportunistic pathogen. An environmental organism, it has no requirement to pass through an animal host to replicate. From the point of view of the bacterium, human infection is a developmental "dead end". Strains which cause disease in humans differ from those causing disease in other animals, by possessing certain genomic islands. It may have the ability to cause disease in humans because of DNA it has acquired from other microorganisms. Its mutation rate is also high, and the organism continues to evolve even after infecting a host.

B. pseudomallei is able to invade cells (it is an intracellular pathogen). It is able to polymerise actin, and to spread from cell to cell, causing cell fusion and the formation of multinucleated giant cells. It possesses a uniquely fusogenic type VI secretion system that is required for cell-cell spread and virulence in mammalian hosts. The bacterium also expresses a toxin called lethal factor 1. B. pseudomalle $i$ is one of the first Proteobacteria to be identified as containing an active type VI secretion system. It is also the only organism identified that contains up to six different type VI secretion systems.

B. pseudomallei is intrinsically resistant to many antimicrobial agents by virtue of its efflux pump mechanism. This mediates resistance to aminoglycosides, tetracyclines, fluoroquinolones, and macrolides.

\section{RISK FACTORS}

Individuals with type 2 diabetes are at increased risk of acquiring melioidosis, a disease caused by Burkholderia pseudomallei infection. Although up to half of melioidosis patients have underlying diabetes, the mechanisms involved in this increased susceptibility are unknown.

\section{MRI BRAIN (PLAIN+CONTRAST) IMAGES OF THE LESION}

A moderately defined lesion with thick enhancing walls noted in the scalp posteriorly with intracranial extension into left parietal lobe. D/d - Necrotic mass - suggested Histopathology correlation.

Absence of normal flow void noted in the adjacent superior sagittal sinus suggestive of thrombosis. 


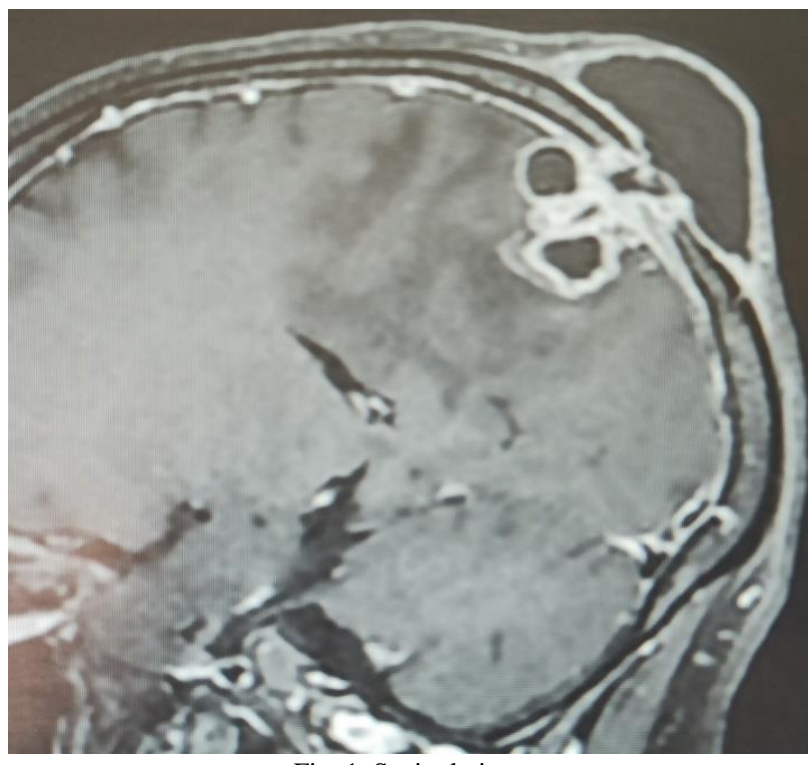

Fig. 1. Sagittal view.

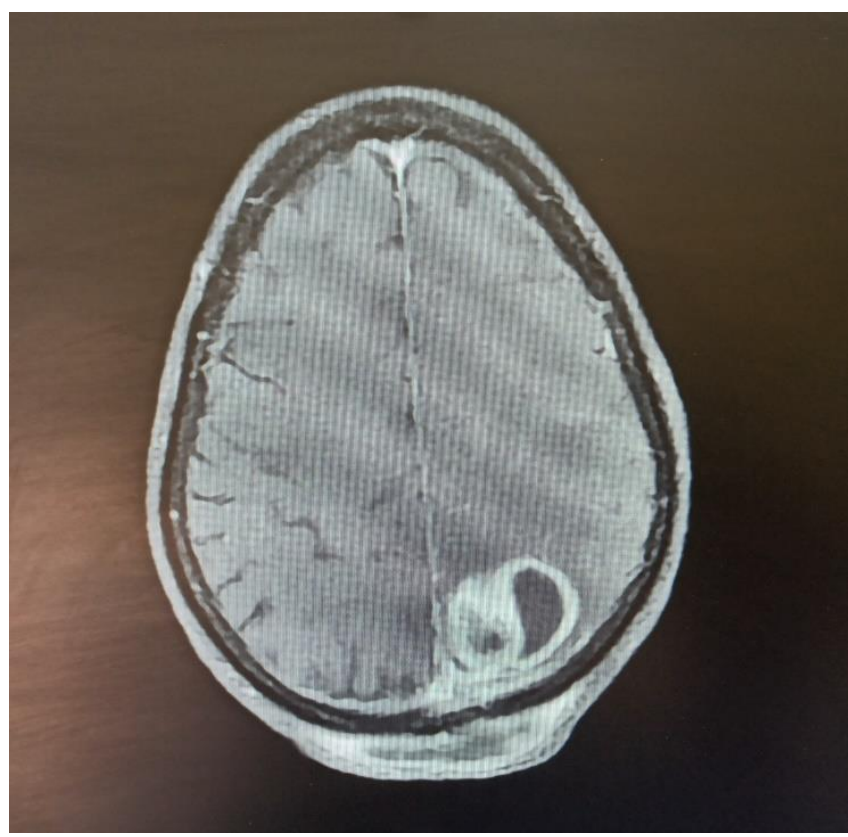

Fig. 2. Axial view.

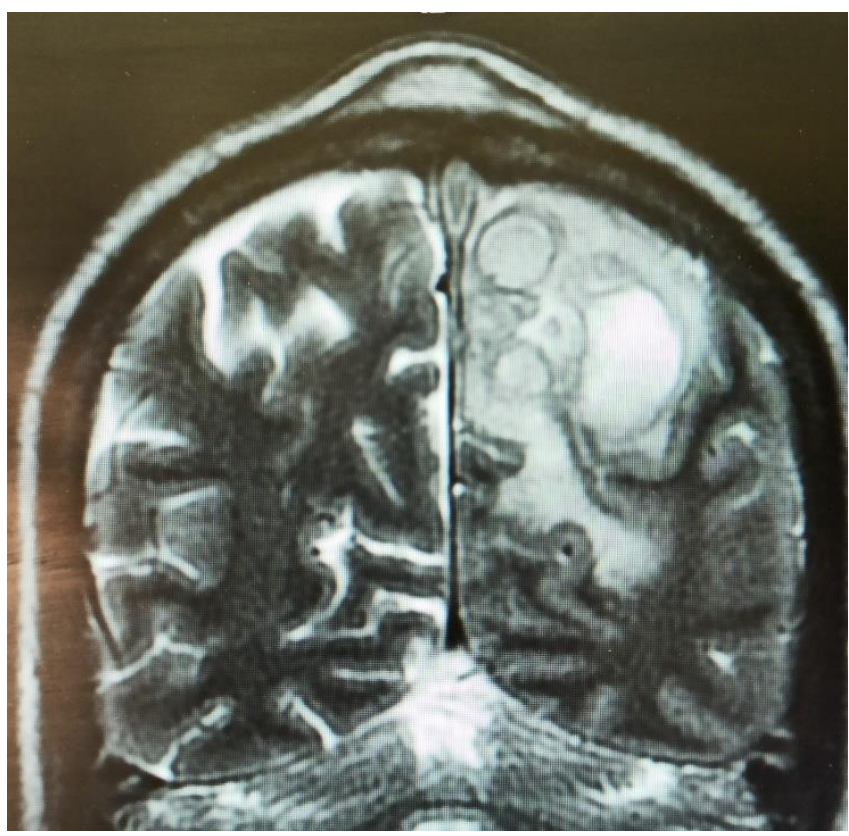

Fig. 3. Coronal view.

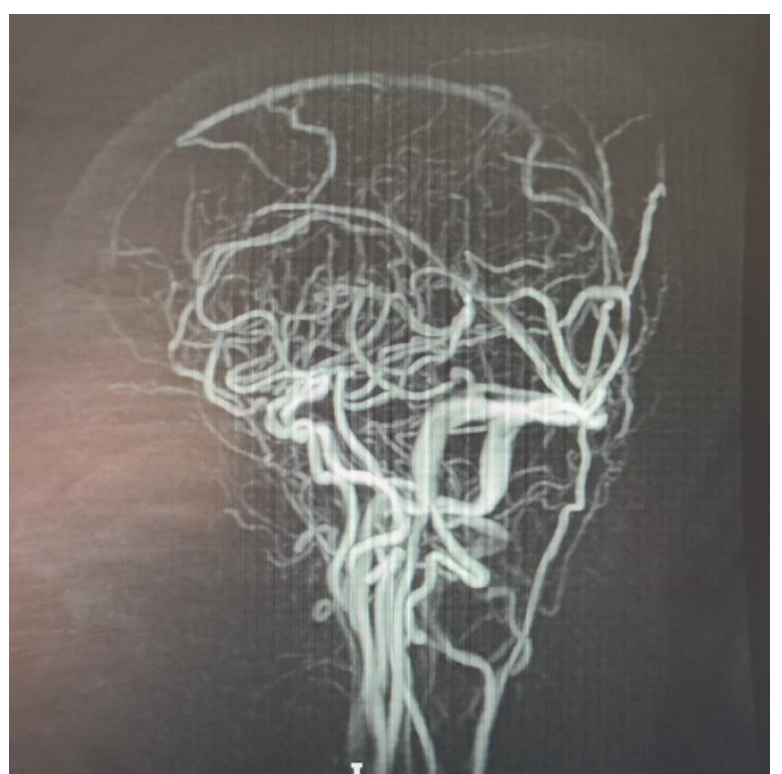

Fig. 4. Mr venogram showing adjacent superior sagittal sinus thrombosis absence of normal flow void- suggestive of thrombosis.
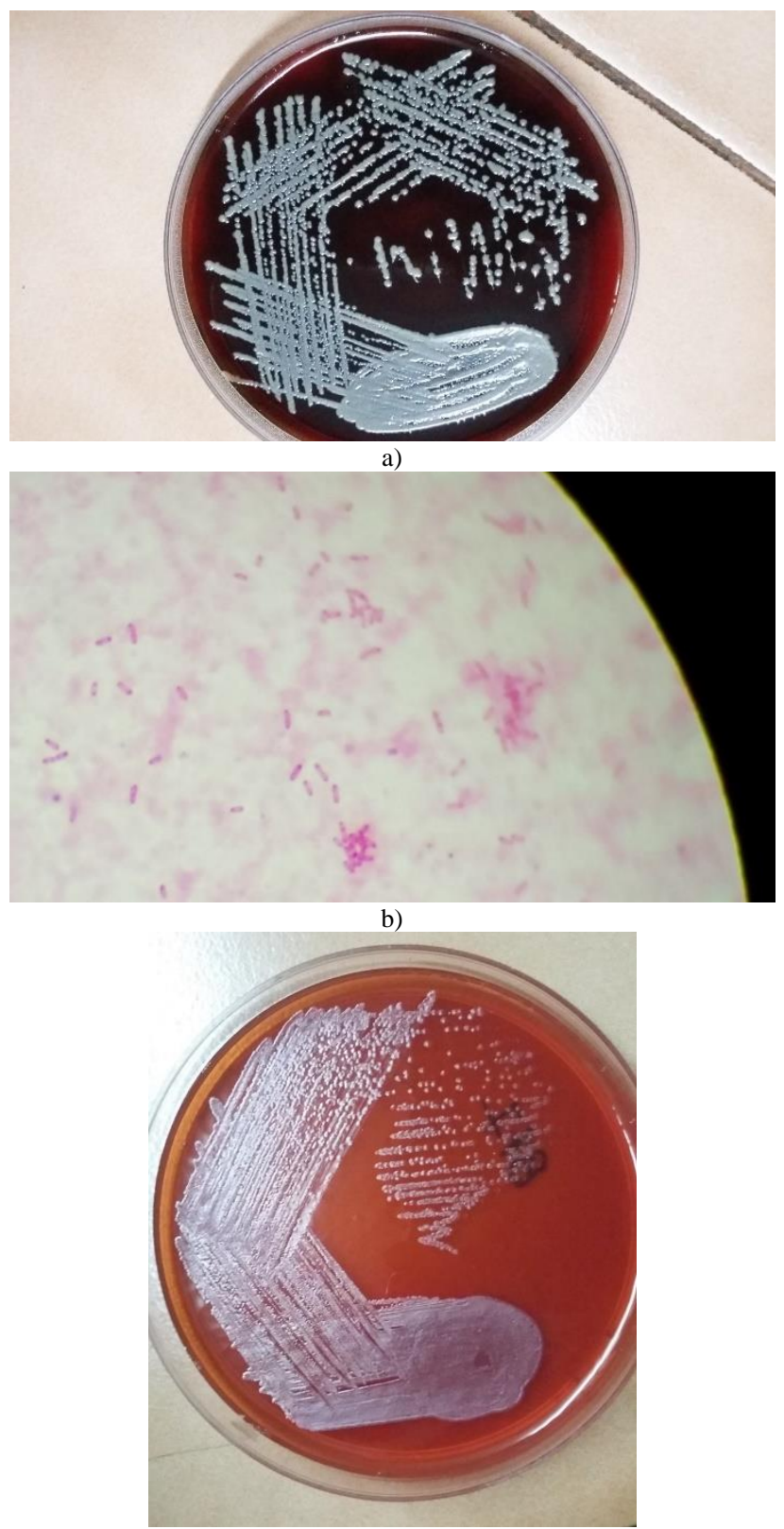

Fig. 5. Culture on blood agar \& MacConkey's agar showed colonies with a slight metallic sheen after $48 \mathrm{~h} \&$ Microscopy. 


\section{REFERENCES}

[1] Hesstvedt L, Reikvam DH, Dunlop O. Neurological melioidosis in Norway presenting with a cerebral abscess. IDCases 2014; 2:16-8.

[2] Vidyalakshmi K, Lipika S, Vishal S, Damodar S, Chakrapani M. Emerging clinico-epidemiological trends in melioidosis: Analysis of 95 cases from western coastal India. Int J Infect Dis 2012; 16:e4917.

[3] Gopalakrishnan R, Sureshkumar D, Thirunarayan MA, Ramasubramanian V. Melioidosis: An emerging infection in India. J Assoc Physicians India 2013; 61:612-4.

[4] Limmathurotsakul D, Chaowagul W, Wongsrikaew P, Narmwong A, Day NP, Peacock SJ. Variable presentation of neurological melioidosis in Northeast Thailand. Am J Trop Med Hyg 2007; 77:118-20.

[5] Hassan MR, Pani SP, Peng NP, Voralu K, Vijayalakshmi N, Mehanderkar $\mathrm{R}$, et al. Incidence, risk factors and clinical epidemiology of melioidosis: A complex socio-ecological emerging infectious disease in the Alor Setar region of Kedah, Malaysia. BMC Infect Dis 2010; 10:302.

[6] Kumar GS, Raj PM, Chacko G, Lalitha MK, Chacko AG, Rajshekhar V. Cranial melioidosis presenting as a mass lesion or osteomyelitis. J Neurosurg 2008; 108:243-7.

[7] Muthusamy KA, Waran V, Puthucheary SD. Spectra of central nervous system melioidosis. J Clin Neurosci 2007; 14:1213-5.

[8] Dance DA, Wuthiekanun V, Naigowit P, White NJ. Identification of Pseudomonas pseudomallei in clinical practice: Use of simple screening tests and API 20NE. J Clin Pathol 1989; 42:645-8.

\section{AUTHOR CONTRIBUTIONS}

1. Conceptualization - Dr. Allen Sibil S (Neurosurgery).

2. Supervision - Dr. Suresh Kumar S (HOD Dept. of Neurosurgery).

3. Writing original draft - Dr.Allen Sibil ( Neurosurgery).

4. Writing- review and editing-Dr. Allen Sibil, Dr. Suresh Kumar S, Dr. Aiswariya Alex (Microbiology). 